\title{
A critical review of the practices of proton daily quality assurance programs
}

\author{
Xiaoning Ding, James E. Younkin, Jiajian Shen, Martin Bues, Wei Liu \\ Department of Radiation Oncology, Mayo Clinic, Phoenix, AZ, USA \\ Contributions: (I) Conception and design: X Ding, W Liu, M Bues; (II) Administrative support: W Liu, M Bues; (III) Provision of study materials or \\ patients: X Ding, JE Younkin, J Shen; (IV) Collection and assembly of data: JE Younkin, J Shen; (V) Data analysis and interpretation: X Ding, JE \\ Younkin, J Shen, W Liu; (VI) Manuscript writing: All authors; (VII) Final approval of manuscript: All authors. \\ Correspondence to: Xiaoning Ding, PhD. Assistant Professor, Department of Radiation Oncology, Mayo Clinic, 5881 E. Mayo Boulevard, Phoenix, AZ \\ 85054, USA. Email: Ding.Xiaoning@mayo.edu.
}

\begin{abstract}
Demand for proton therapy (PT) continues to grow due to its dosimetric advantages over conventional radiotherapy. New PT facilities being constructed to meet this demand will need quality assurance (QA) programs to ensure that treatments are delivered safely and accurately. However, in contrast to conventional radiotherapy, proton QA practices are constantly evolving and few commercial solutions are available. As a result, QA programs at most operational proton facilities rely on a variety of in-house developed hardware and software. An important part of these QA programs is proton daily QA, which verifies clinically-acceptable proton delivery system operation each morning before starting patient treatment. In this review article, we summarize current proton daily QA practices by providing a brief introduction to PT, describing proton delivery techniques and their particular QA requirements, and then reviewing implementations of several proton daily QA programs. Although the QA instrumentation is quite heterogeneous, the literature shows that the dosimetric daily QA results among proton facilities are comparable. We also present a typical set of proton daily QA data from our institution that includes output, range, and spot position measurements. Based on the literature review and our institutional experience, we make recommendations for future proton daily QA programs.
\end{abstract}

Keywords: Proton therapy (PT); pencil beam scanning (PBS); daily quality assurance (daily QA); range verification; spot position verification

Received: 17 May 2021; Accepted: 01 November 2021; Published: 30 December 2021.

doi: $10.21037 /$ tro-21-11

View this article at: https://dx.doi.org/10.21037/tro-21-11

\section{Introduction}

It is now well known that proton therapy (PT) can deliver superior dose distributions to tumors and organs at risk (OARs) (1). Historically, Wilson first proposed using the finite range of a proton beam to treat tumors adjacent to healthy tissues in 1946 (2). The advantages of PT over conventional X-ray based radiotherapy include elimination of exit dose and reduction of proximal dose. These advantages enable PT to reduce dose to surrounding OARs and escalate the dose to tumors, which in theory should reduce the chance of toxicities while increasing the probability of local tumor control. PT is particularly attractive for some tumor types and sites, such as head-andneck cancers and for pediatric cancer patients. In the last decade, the advantages of PT have been further improved through the use of scanning-beam technology, which makes it possible to deliver intensity modulated PT (IMPT) $(3,4)$. For these reasons, the number of PT centers has grown rapidly over the last two decades. As of today, two hundred thousand cancer patients have been treated with PT and there are over one hundred operational PT facilities (1) worldwide.

Comprehensive quality assurance (QA) programs are essential to ensure that patients receive safe and effective 
radiotherapy treatments. QA programs consist of several procedures performed with different frequency (daily, weekly, monthly, annual, or patient-specific). Daily QA is performed every day before the start of patient treatment. It is a final check ensuring that all major components of the treatment delivery system are functioning properly. PT centers developing a proton daily QA program will face a number of additional challenges compared to conventional radiotherapy. First, there were no standard guidelines for proton QA until the American Association of Physicists in Medicine (AAPM) Task Group 224 report (5) was published in 2019. Second, there are very few commercially available solutions for proton daily QA. Third, complex PT systems require a comprehensive list of QA tests that must be performed efficiently due to the higher costs of beam-on time compared to conventional linear accelerators. Because of these additional challenges, establishing a proton daily QA program demands substantial efforts in both design and implementation.

In this manuscript, we review and summarize the practice of proton daily QA. A brief introduction to PT and different proton delivery techniques is given from the perspective of proton daily QA (section "Accelerator and beam delivery techniques") followed by an overview of various proton daily QA requirements and several daily QA programs (section "Content and implementation of proton daily QA"), dosimetric results from one selected daily QA procedure (section "Content and implementation of proton daily QA"), and discussions and recommendations (section "Discussion and recommendations").

\section{Accelerator and beam delivery techniques}

Typical cancer treatments utilize proton energies in the $\sim 70-250 \mathrm{MeV}$ range. The penetration depths in water (i.e., proton range) corresponding to these energies are between 4 and $38 \mathrm{~cm}$ (see NIST PSTAR database, https://physics. nist.gov/PhysRefData/Star/Text/PSTAR.html). It should be noted that proton energy and proton range in water are often used interchangeably in the PT literature. An accelerator provides narrow proton beamlets at the energies that are required to treat the target volume. These beamlets are transported to the treatment room, where a nozzle spreads the narrow beamlets and sometimes modifies the beamlet energy to produce a proton dose distribution that conforms to the treatment target. Since the delivered dose distributions are highly conformal, PT requires an imageguided radiotherapy (IGRT) system [e.g., kilovoltage $(\mathrm{kV})$
X-ray, cone-beam CT (CBCT), etc.] to precisely establish patient treatment position (6).

\section{Accelerator}

Clinical proton delivery systems accelerate protons to the clinical energy range using either cyclotrons or synchrotrons $(7,8)$. Cyclotrons output protons at a single fixed energy, the maximum energy of the proton delivery system, and use energy degraders and energy selection systems to reduce the proton beam energy as required for treatment. Cyclotrons are simpler to operate, more compact in size, and can deliver higher dose rates. On the other hand, synchrotron-based proton delivery systems can accelerate protons to each of the discrete energies required for treatment, thereby eliminating the need for energy degraders. From the perspective of the machine QA program, cyclotron-based and synchrotron-based proton delivery systems have identical QA requirements. All other things being equal, the same QA procedures can be used for either accelerator type.

\section{Beam delivery techniques}

The three beam delivery techniques used in PT are double scattering (DS) (9), uniform scanning (US) (10), and pencil beam scanning (PBS) (11). Initially, the DS technique was the only delivery technique available. However, PBS has now become the favored technique for newly-constructed proton facilities due to its superior dose conformality $(12,13)$ and operational simplicity. Most proton facilities currently in operation either use PBS exclusively or have at least one treatment room dedicated to PBS.

While machine QA requirements are independent of accelerator type, they strongly depend on the delivery technique. DS and US deliver a uniform field with a spread-out Bragg peak (SOBP), and therefore lateral dose uniformity and SOBP width are important QA parameters for these delivery techniques. However, PBS delivers a sequence of proton beamlets, so the position, width, and range of these beamlets replace SOBP width and uniformity as the fundamental QA parameters. In the following sections, we provide details of PT delivery techniques that are necessary to understand their particular daily QA requirements.

\section{DS}

In a DS delivery system, the narrow proton beam entering 
the nozzle is spread out laterally by two scatterers (9). The first scatter broadens the beam while the second scatter further broadens the beam and flattens the fluence profile to achieve uniform intensity and range across the field. Since these two scatterers are placed in the beam path, they also reduce the proton range. Thicker scatterers are required to produce a bigger field, so larger field sizes require more scattering, which reduces proton range and dose rate. A rotating range modulation wheel (RMW) with variable thickness that rotates at several hundred revolutions per minute is used to modulate the energy and intensity of multiple Bragg peaks to produce a uniform SOBP (9). Multiple scatterers and RMWs are required to generate irradiation fields with different field sizes, ranges, and modulations, and the computer control system maintains a library of beam parameters for each of the hardware combinations.

The DS technique uses apertures and range compensators (RCs) to achieve a dose distribution that is conformal to the target volume laterally and distally, respectively (9). The aperture shapes the field so that it conforms to the maximum lateral extent of the beam's-eye-view of the target volume. Apertures are field-specific and commonly made of brass. The RCs are used to shape the dose distribution to be conformal to the distal edge of the target volume. RCs are also field-specific and are commonly made of acrylic. While the shape of the RC reduces the range by variable amounts across the field, the modulation width of the SOBP is fixed. Therefore, the resulting dose distribution conforms well to the distal end of the target volume but is not conformal on the proximal side, which can result in high doses to a large volume of proximal normal tissue.

\section{US}

In a US delivery system, scanning magnets in the nozzle deflect the narrow incoming beam to generate a laterally uniform proton intensity, thereby eliminating the need for using scatters to broaden the narrow beam (14). The US system uses the method of energy layer stacking to produce the desired SOBPs. The layer switching is done with range shifters, RMWs, or a combination of both. Similarly to the DS technique, field-specific apertures and RCs are required to produce the desired dose distribution. The advantage of US over DS is that no scatterer is needed. Because there is less energy loss due to no scatter along in the nozzle, US can achieve higher ranges and greater accelerated proton usage efficiency compared to DS.

US has never been a popular choice in PT. Today only a handful of proton facilities still have treatment rooms dedicated to the US technique. The range increase offered by US over DS is on the order of several centimeters in water, but this small advantage comes at the cost of introducing the interplay effect (15-18), which is caused by the relative motion between a moving tumor and a scanned beam. More importantly, the PBS delivery technique was developed at about the same time as the US technique, and the advantages of PBS over US quickly squeezed the US technique out of the market.

\section{PBS}

In a PBS delivery system, the narrow proton beam (often called a beamlet or spot) is deflected in two transverse directions by scanning magnets (19). The proton beamlets are delivered in a spot-by-spot manner within one energy layer and energy layer by energy layer in depth. The beam is usually delivered from the most distal layer (i.e., the highest energy) to the most proximal layer (i.e., the lowest energy). The advantages that PBS offers are threefold: it has superior dose conformality, greater flexibility in terms of dose delivery, and simplified treatment operation that practically eliminates patient-specific hardware. Since spots are delivered independently, the spot intensities can vary between different spots and energy layers. This makes it possible to use spot-weighted delivery techniques that can significantly improve dose conformality $(12,13)$. It also makes possible a form of IMPT in which the spot intensities of multiple fields are optimized simultaneously for conformality and improved normal tissue protection. This high degree of freedom in dose delivery offers the flexibility to deliver a similar dose distribution with many different beam angles and spot intensity solutions. PBS does not usually require the use of field-specific aperture and RC. In fact, aperture and RC are seldom used in PBS except in a few cases to treat very small tumors (such as an ocular melanoma) in a stereotactic setting (20). This greatly simplifies the treatment delivery process. Due to the advantages mentioned above, it is expected that virtually all PT facilities will provide PBS exclusively in the future.

Unfortunately, PT is sensitive to proton beam range uncertainties, patient setup uncertainties, and breathing motion-induced interplay effects (16-18). However by using robust optimization in PBS treatment planning, spot intensity solutions with less sensitivity to beam range and patient setup uncertainties can be automatically selected by the optimizer to mitigate the influence of uncertainties (21-38). Motion-related impact such as interplay effects 
can be further mitigated by $4 \mathrm{D}$ robust optimization (39-44). Plan robustness evaluation as a QA of the impact of uncertainties has been implemented in some commercial proton TPSs and routinely used in most proton centers (37,45-48). Interplay effect evaluation as QA for the impact of motion has also been routinely used in some proton centers (49-53).

\section{Imaging systems}

PT requires IGRT in order to take full advantage of the superior dose distributions (6). Historically, PT was one of the first radiation modalities to employ in-room imaging for patient setup (54). $\mathrm{kV}$ imaging systems have been routinely used in proton facilities. Typically a set of two-dimensional $\mathrm{kV}$ images is compared to digitally reconstructed radiographs (DRRs) produced from the planning CTs to align the patient prior to treatment. The $\mathrm{X}$-ray tubes may be mounted in the gantry, in the floor, or in the ceiling. However, PT has lagged behind conventional radiotherapy in volumetric image guidance. Gantry-mounted CBCT was not commercially available for PT until 2014. The delay of volumetric imaging guidance was caused by the cost of incorporating CBCT into the proton gantry and the quality of CBCT (6). Today, all major PT vendors offer CBCT integrated within their treatment units (55). From the perspective of proton QA, the recommendations for CBCT in conventional radiotherapy can be directly applied to PT. As stated in TG-224 (5), the required procedures for CBCT daily QA should follow the guidance given by other published AAPM documents (56-58).

\section{Content and implementation of proton daily QA}

QA procedures for conventional radiotherapy are wellestablished. TG-40 (59), which was published in 1994, has been the standard reference document of QA programs for medical linear accelerators (linacs). TG-142 (57) was published in 2009 as an update of TG-40 in order to incorporate recent technological advances. These two documents are well-accepted guidelines for QA programs in conventional radiotherapy. In contrast, QA guidelines for proton radiotherapy are less well-established. The first hospital-based PT facility in USA (i.e., the Proton Treatment Center at Loma Linda University Medical Center) was opened in 1990 (60). But it took almost 30 years for the proton community to publish the first QA document, TG-224 (5). This delay was caused by both economic and technical factors. PT is much more expensive than conventional radiotherapy, which has slowed down the pace of PT deployment. At the same time, PT technology has been continuously evolving. It takes time for proton machine vendors to incorporate new technologies and for proton facilities to adopt them. Therefore, the daily QA programs in proton facilities worldwide today are mostly inhouse developed solutions.

A comprehensive daily QA program requires a delicate balance between the QA contents and the execution time. To balance these two competing factors, the proton daily QA program has to be optimized for simplicity and efficiency. Proton daily QA should take no more than 30 minutes to finish but be able to verify that the PT system has been maintained at the state of commissioning within the tolerance. Unlike the linacs used for conventional therapy, PT facilities usually have multiple treatment rooms that receive beams from a single accelerator, and the proton beam can only be delivered to one treatment room at a time. Therefore, beam-on time is a scarcer resource in PT than conventional radiotherapy. Daily QA programs requiring several field deliveries in multiple treatment rooms can create long beam waiting times that can significantly delay the start of treatment.

Another challenge is to decide who performs the daily QA. The daily QA procedure is designed by qualified medical physicists (QMPs) and usually executed by QMPs initially when the facility is opened. After the procedure is streamlined and it has matured, the work can be transferred to medical physicist assistants (MPAs) or trained radiation therapists. From the perspective of clinical operation, it is advantageous for therapists to perform daily QA since they can start patient treatment immediately after test results have been reviewed by QMPs. However, therapists usually have less professional experience with QA instrument operation. If therapists are assigned to perform daily QA, special attention should be paid to the simplicity of the daily QA procedure and the provision of adequate training.

\section{Components of daily $Q A$ program}

TG-224 provides comprehensive QA guidelines for each PT delivery modality, including DS, US, and PBS. The report serves as supplement to the well-established QA guidelines for photon and electron machines, such as TG-142 and TG40. Although comprehensive QA guidelines for radiotherapy should also include patient-specific QA (PSQA), TG-224 focuses on periodic machine QA exclusively. However, there are many publications that describe PSQA in PT for 
Table 1 Daily QA procedures for PT

\begin{tabular}{|c|c|c|c|}
\hline Daily QA test & \multicolumn{3}{|c|}{ Tolerances } \\
\hline \multicolumn{4}{|l|}{ Dosimetry } \\
\hline Output constancy & $\pm 3 \%$ & $\pm 3 \%$ & $\pm 3 \%$ \\
\hline \multicolumn{4}{|l|}{ Depth verification } \\
\hline Proximal & $\pm 2 \mathrm{~mm}$ & $\pm 2 \mathrm{~mm}$ & \\
\hline SOBP width & $\pm 2 \% / \pm 2 \mathrm{~mm}$ & $\pm 2 \% / \pm 2 \mathrm{~mm}$ & \\
\hline Spot position & & & $\pm 2 \mathrm{~mm} / \pm 1 \mathrm{~mm}$ \\
\hline \multicolumn{4}{|l|}{ Mechanical (all delivery system) } \\
\hline \multicolumn{4}{|l|}{ Imaging } \\
\hline $\mathrm{X}$-ray isocenter vs. laser isocenter & & $\pm 2 \mathrm{~mm}$ & \\
\hline $\mathrm{X}$-ray isocenter vs. beam isocenter & & $\pm 1 \mathrm{~mm}$ & \\
\hline СBCT & & Functional & \\
\hline
\end{tabular}

QA, quality assurance; PT, proton therapy; DS, double scattering; US, uniform scanning; PBS, pencil beam scanning; SOBP, spread-out Bragg peak; CBCT, cone-beam CT.

the interested reader (61-63). TG-224 identifies the proton beam parameters that are typically checked and contains 4 tables listing the recommended QA procedures for daily, weekly, monthly, and annual QA. These procedures heavily depend on proton delivery modalities. Table 1 lists the recommended evaluation metrics and their tolerances for proton daily QA. The tests performed during proton daily QA can be categorized into safety, mechanical, imaging, and dosimetry checks. Of these, only the dosimetry tests change with delivery technique. Table 1 is included here for reference. Note that the safety section are not included for brevity.

The proton daily QA dosimetry tests include checks of dose output and proton range. For the output measurement, using ionization chambers with large sensitive volumes results in more stable measurements by collecting greater total charge and averaging over a larger volume. The measurement point should be at a position with low dose gradient to mitigate the impact of setup uncertainty. However, there are differences between DS/US and PBS for some beam parameters. As explained above, DS and US deliver uniform fields over a large volume. Therefore, their QA procedures include verify the dose at the proximal and distal edges of the SOBP (5). In order words, both distal range and proximal range should be measured, in order to check the constancy of the generated SOBPs. In contrast, the QA procedure for PBS focuses on checking the characteristics of individual beamlets, such as range, spot position and size (5).

The tolerances given in Table 1 of TG- 224 are $\pm 3 \%$ for output constancy and \pm 1 or $\pm 2 \mathrm{~mm}$ for range constancy depending on the delivery technique and measurement position. For PBS spot position, the tolerance is $\pm 2 \mathrm{~mm}$ from the perspective of imaging system (absolute), and $\pm 1 \mathrm{~mm}$ from the perspective of spot centrioles relative to each other (relative). The output tolerance is adopted from TG-40 and is based on the principle that dose delivered to patient should be within $\pm 5 \%$ of the prescribed dose.

\section{Implementations of daily $Q A$ programs}

The potential market for proton daily QA equipment is currently small and the requirements for a commercial proton daily QA device are challenging due to the delivery system heterogeneity and the lack of daily QA procedure standardization until recently. Thus, the products 


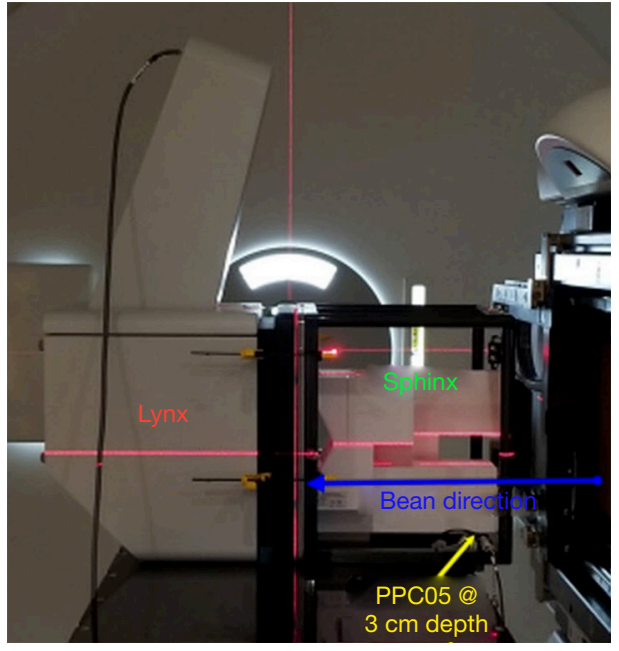

Figure 1 Lynx and Sphinx set up on treatment couch. The proton gantry is horizontal.

commercially available for proton daily QA are very limited. As a result, many PT clinics have taken a hybrid approach, using equipment designed for linac daily QA together with in-house developed hardware and software.

\section{Commercial solutions}

There were no dedicated commercial solutions for proton daily QA until 2018. So far the only two commercial solutions are from IBA Dosimetry (Schwarzenbruck, Germany), which offers Sphinx/Lynx and Sphinx Compact $(64,65)$. Both solutions are designed to check the constancy of PBS beam characteristics. The Sphinx/Lynx system consists of two components-Sphinx is the dosimetric phantom and Lynx is the radiation detector. The Sphinx Compact is a newer version of Sphinx/Lynx. In Sphinx Compact, Lynx was replaced by a flat-panel detector to make the system more compact and modular. Both solutions have been used in operational proton facilities for PBS daily QA, and it has been reported they both can fulfill the daily QA requirements recommended by TG-224 $(64,65)$. Figure 1, reprinted from the literature, shows the setup of Sphinx and Lynx (64). As shown in the figure, Sphinx and Lynx are coupled mechanically and placed on a treatment couch. Figure 2 is another reprinted figure that shows the Sphinx device. Sphinx contains 4 wedges of different thickness, as well as a carbon fiber frame to hold the wedges and water-equivalent polystyrene (RW3) blocks. Each wedge is irradiated by the proton beams in line pattern with 4 energies (106, 145, 172 and $221 \mathrm{MeV}$ ) for range constancy verification. A dedicated RW3 block contains a notch to hold a removable parallel-plate chamber (IBA PPC05) for dose output constancy verification. Lynx consists of a scintillating screen and a CCD camera contained in a compact box. The line patterns produced by wedges on the screen are interpolated to obtain the proximal and distal ranges. Individual proton beamlets with different energies are delivered to the screen for the verification of spot positions and spot sizes.

\section{Hybrid approach}

As mentioned above, there were no standard guidelines for proton daily QA until the TG-224 report was published in 2019, and no dedicated equipment was commercially available until 2018. As a result, the majority of operational proton facilities use in-house developed daily QA programs. As one might expect, proton facilities belonging to large research hospitals typically develop more complex and comprehensive QA solutions, while smaller or primarily clinically-focused proton centers tend to develop simpler solutions to maximize clinical efficiency.

Actis et al. described a daily QA solution used for the PBS gantry at Paul Scherrer Institute (PSI) (66). This solution includes very sophisticated hardware and software. The hardware (i.e., the dosmetric phantom) consists of a multi-leaf ionization chamber (MLIC) for energy constancy verification, strip chambers for the measurement of spot position and spot size, ionization chamber holders for dose output constancy verification, and scintillating screens for checking proton beamlets. Figure 3 is a reprinted figure from the published literature that shows this dosmetric phantom (66). The software executes QA procedures automatically and stores the measured data in a database for trend analysis. The beam parameters checked during daily QA include dose output, ranges, and spot positions. The daily QA program is comprehensive enough that most weekly and monthly checks are included, as well as so highly efficient that it takes only 20 minutes to complete. However, this approach demands a high level of expertise in both physics and engineering, which makes it a difficult approach for other facilities to adopt.

The University of Texas M. D. Anderson Cancer Center treats cancer patients using both DS and PBS techniques, and they use different QA procedures for each of the two techniques. The DS daily QA procedure uses a cylindrical Farmer-type ionization chamber and acrylic blocks to measure dose output, ranges, and widths of SOBPs (67). The PBS daily QA procedure measures 

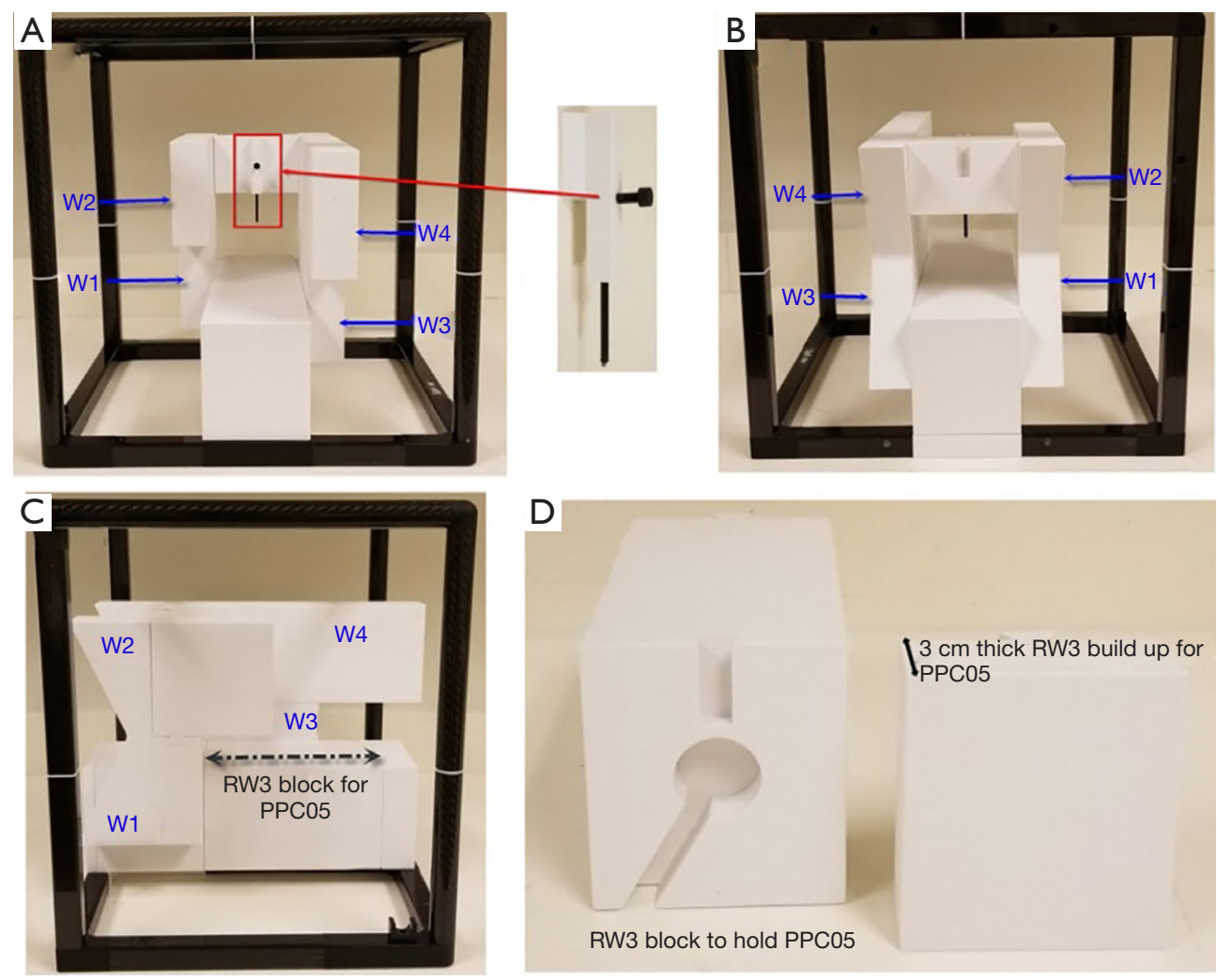

Figure 2 The Sphinx phantom has a carbon frame with dimension of $540 \mathrm{~mm} \times 400 \mathrm{~mm} \times 400 \mathrm{~mm}$. The carbon frame contains the markers for verification of laser alignment. (A) The Sphinx device is shown with fiducial insert for X-ray vs. proton beam coincidence. (A-C) The RW3 blocks with wedges (W1, W2, W3 and W4) are shown in front (A), back (B), and side (C) views. The W1, W2, W3 and W4 are used to measure the ranges, widths, and distal-fall-off of energies 106, 145, 172 and $221 \mathrm{MeV}$, respectively. (D) The RW3 block is shown with the cutout for the PPC05 parallel plate chamber as well as $3 \mathrm{~cm}$ thickness buildup that is placed in front of the chamber.

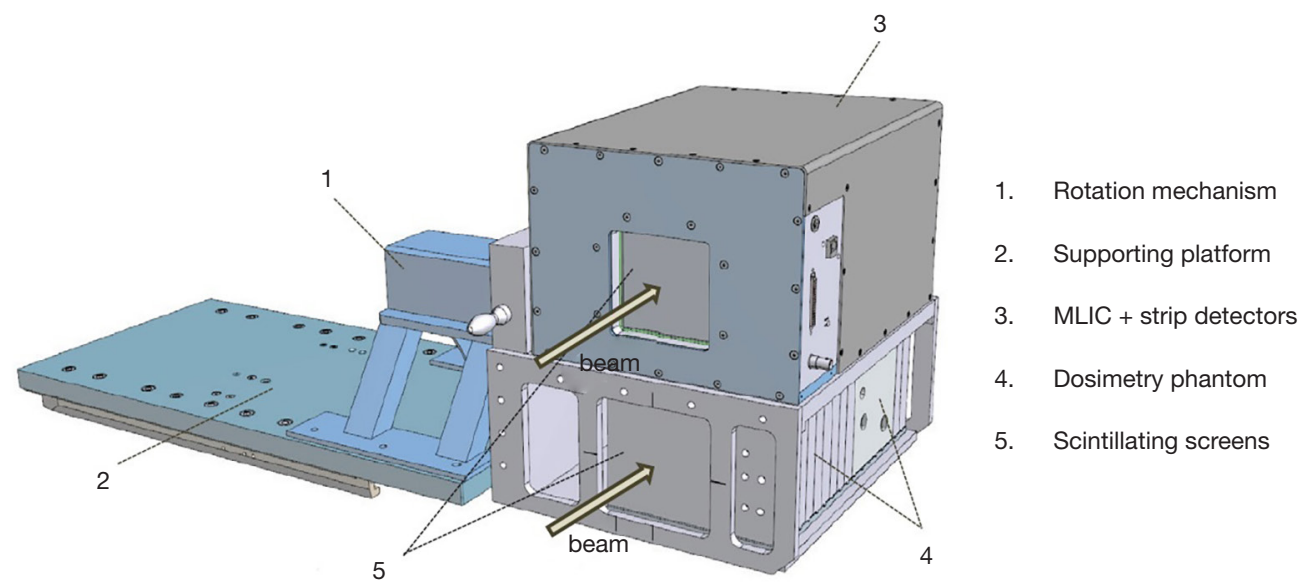

Figure 3 The daily QA hardware at PSI for the verification of spot positions, ranges, and dose output. An aluminum box (no. 3) contains MLIC and two-step chambers for the verifications of range and spot position. The phantom (no. 4) consists of a PMMA block with two embedded ionization chambers for proton dose output verification. Two scintillating screens (no. 5) are fixed to the phantom, which allows a visual check on the size and shape of proton spots. QA, quality assurance; PSI, Paul Scherrer Institute; MLIC, multi-leaf ionization chamber; PMMA, polymethyl methacrylate. 

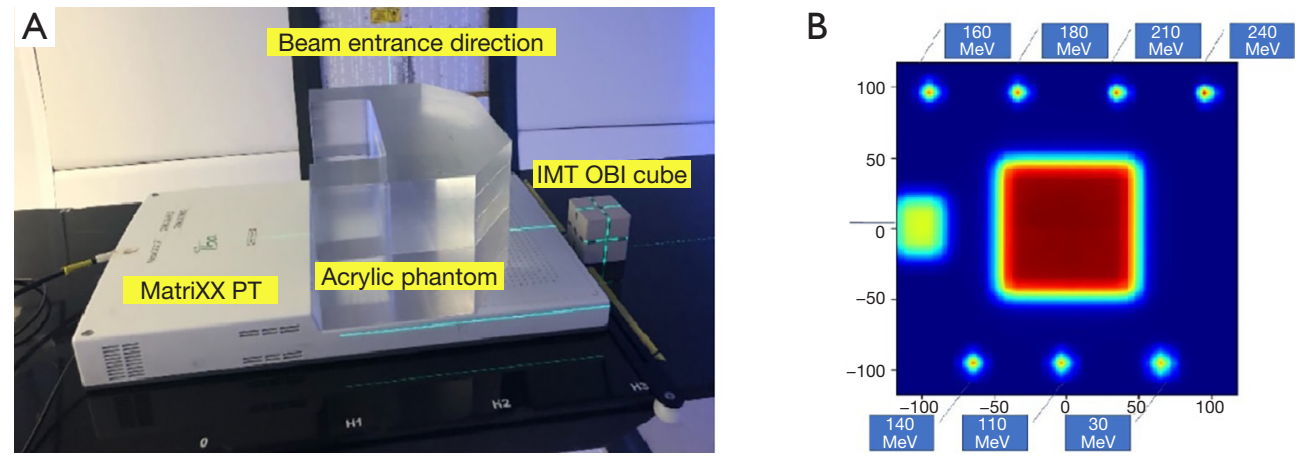

Figure 4 The typical setup image of using MatriXX PT for daily QA at New York Proton Center. (A) Hardware placement layout and (B) the dose distribution measured by the MatriXX PT during the proton daily QA. PT, proton therapy; QA, quality assurance.

spot positions as well as dose output and ranges using five parallel-plate ionization chambers arranged in a cross with $10 \mathrm{~cm}$ separation (68). The daily QA practice at the MD Anderson PT center is a good example of a proton daily QA program in which the content and implementation depend substantially on the available delivery techniques.

Some PT centers have adapted the MatriXX PT (IBA Dosimetry, Schwarzenbruck, Germany) for use in proton daily QA $(69,70)$. MatriXX PT is a $2 \mathrm{D}$ array of parallel plate ionization chambers. It has 1020 air-vented ion chambers arranged in $32 \times 32$ grid, with $7.6 \mathrm{~mm}$ spacing between chambers. It can provide $2 \mathrm{D}$ absolute dose distribution at various depths when it is used with plastic phantom slabs. These capabilities have made the MatriXX PT useful for proton PSQA (61). With an in-house developed phantom, MatriXX PT can also be used for proton daily QA dosimetry measurement. Shi et al. reported using MatriXX PT and an U-shaped phantom to check PBS beam parameters during daily QA (70). MyQA ${ }^{\mathrm{TM}}$ software (IBA dosimetry, Schwarzenbruck, Germany) was used for logging the dosimetry QA data. In-house developed software was used to provide immediate feedback during daily QA and trend analysis later. Figure 4 includes two reproduced figures from the published literature to show the hardware layout and the expected MatriXX PT measured image. The acrylic phantom was used with MatriXX PT together, searved as a buildup for SOBP. The on-board-imaging (OBI) cube was used for imaging QA purposes. A test pattern was designed to test the beam parameter consistency including output, range, spot position, and spot size.

The Daily QA ${ }^{\mathrm{TM}} 3$ (DQA-3, Sun Nuclear Inc., Melbourne, FL, USA) is a popular commercial device that has been employed for proton daily QA (71-73). The DQA3 is designed for linac daily QA and contains 13 vented ionization chambers (1 thimble chamber and 12 parallelplate chambers) as well as 12 diodes. These detectors are arranged on a $24 \mathrm{~cm} \times 26 \mathrm{~cm}$ flat panel. The 4 electron energy chambers $\left(\mathrm{e}^{\mathrm{TL}}, \mathrm{e}^{\mathrm{TR}}, \mathrm{e}^{\mathrm{BL}}\right.$ and $\mathrm{e}^{\mathrm{BR}}$ ) have different amount of intrinsic buildups ranging from 0.2 to $4.3 \mathrm{~g} / \mathrm{cm}^{2}$. These differences in buildup provide a simple way to monitor proton range constancy. By combining nonuniform field patterns and in-house developed phantoms, the DQA-3 has been converted to an effective tool for proton daily QA in many facilities. Ding et al. were the first to demonstrate the use of DQA-3 for proton daily QA (71), showing how to use the device to check dose output, range, and symmetry for a US proton facility. Figure 5 includes four reproduced figures from this study to show the concept of range constancy check (71). As shown in the figures, a DQA-3 is attached to a mechanical jig, which can be locked on a treatment couch by an indexed bar. Three compensators that can be mounted to proton snout are used for three range checks (10, 16 and $24 \mathrm{~cm})$. The thicknesses of the compensators are carefully chosen to pull the beams back that set the chambers of $\mathrm{e}^{\mathrm{TR}}$ and $\mathrm{e}^{\mathrm{BL}}$ at the region close to the middle of SOBP, while the chambers of $\mathrm{e}^{\mathrm{TL}}$ and $e^{B R}$ are positioned at the distal edge of the SOBP. The $e^{T L}$ and $\mathrm{e}^{\mathrm{BR}}$ measurements are very sensitive to proton energy fluctuation, while the measurements from $\mathrm{e}^{\mathrm{TR}}$ and $\mathrm{e}^{\mathrm{BL}}$ are not. Therefore the ratios of $\mathrm{e}^{\mathrm{TL}}$ and $\mathrm{e}^{\mathrm{BR}}$ measurements to $\mathrm{e}^{\mathrm{TR}}$ and $\mathrm{e}^{\mathrm{BL}}$ measurements were used for the range constancy check.

Lambert $e t a l$. further extended the use of DQA-3 to monitor PBS beamlets characteristics (72). The DQA-3 diodes were used to monitor the spot position and spot size, as well as output, ranges, etc. Younkin $e t$ al. continued the improvements using the combination of nonuniform proton field and a two-step wedge phantom (see Figure 6) (73). 

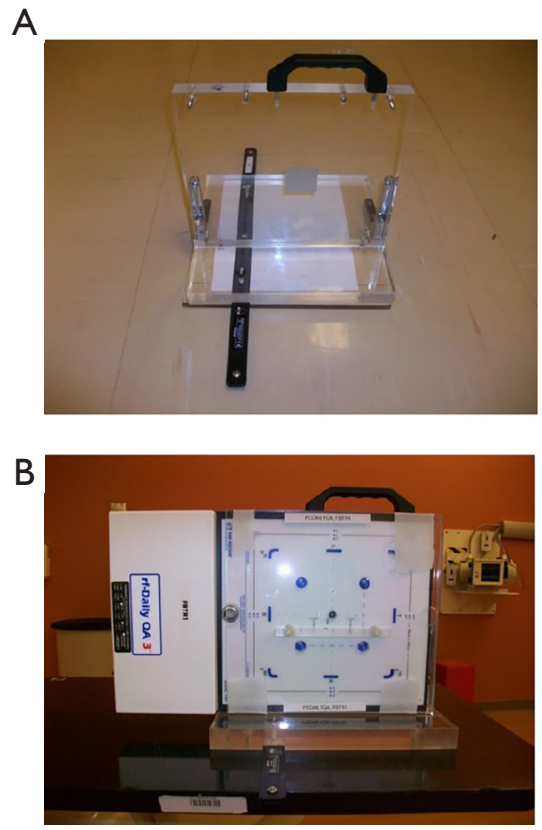

C
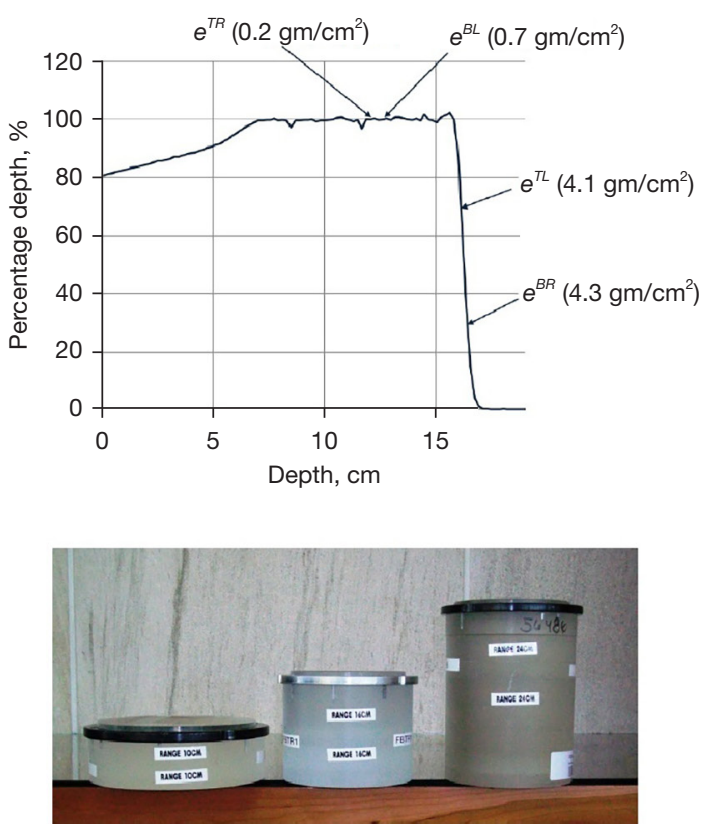

Figure 5 An initial set of in-house developed apparatus used for proton daily QA of a PT system using the US delivery technique and the technique used to measure proton range. (A) The mechanical jig consists of two pieces of acrylic plates with index bar attached onto the bottom plate. Four metal screws on the corners of the vertical plate secure the DQA-3 device. (B) The electron buildups of the four electron energy chambers and their positions on the PDD curve of a proton beam with a range of $16 \mathrm{~cm}$ and a modulation of $10 \mathrm{~cm}$. Chambers of $\mathrm{e}^{\mathrm{TL}}$ and $\mathrm{e}^{\mathrm{BR}}$ are used to collect charges at the distal falloff region while chambers of $\mathrm{e}^{\mathrm{TR}}$ and $\mathrm{e}^{\mathrm{BL}}$ are used to collect charges at the region close to the middle of SOBP. (C) The DQA-3 in the measurement position is sandwiched between the vertical plate and an acrylic imaging phantom (the small plastic bar with three fiducial markers that is attached to the center of the panel). The CAX chamber is indicated by a dark circle on the panel and the four surrounding electron energy chambers are indicated by blue circles. (D) Three compensators with appropriate thicknesses are used for three beam ranges (10, 16 and $24 \mathrm{~cm}$ ). QA, quality assurance; PT, proton therapy; PDD, percentage depth dose; SOBP, spread-out Bragg peak; CAX, central axis.

This setup allows all dosimetric tests to be performed using a single field, which eliminates problematic beam waiting time from earlier procedures. The program developed by Younkin et al. is highly efficient and comprehensive. It takes only 10 minutes for a therapist to perform daily QA including safety, mechanical, imaging, and dosimetry checks for a PBS facility. The dosimetric checks include output constancy, range constancy for two energies, and four spot position checks. Another unique feature is the operational simplicity. After receiving proper training, the therapist who performs patient treatment can perform daily QA checks. This staff arrangement greatly streamlines clinical workflows.

\section{Dosimetric results from $D Q A-3$ based proton daily $Q A$}

The proton daily QA dosimetric results from published literatures $(64,66,70,71,73)$ show consistent results across five proton facilities. In general, measurement variation is within $\pm 1 \%$ for output deviation, within $\pm 0.5 \mathrm{~mm}$ for range deviation, and within $\pm 1 \mathrm{~mm}$ for beamlet spot position deviation. The total execution time is usually less than half an hour. These results reflect the fact that these PT systems are relatively stable and that the reported proton daily QA programs are accurate and efficient.

The PT center at Mayo Clinic Arizona has been operational for cancer treatment since March 2016. The proton delivery system (Hitachi ProBeatV) has one synchrotron accelerator and four gantry treatment rooms dedicated to the PBS technique. In addition, there is an experimental fixed beam room, which is not used clinically or included in daily QA. Mayo Clinic Arizona currently uses the daily QA procedure described in Younkin et al. and the setup shown in Figure 6 involving the DQA-3, the 

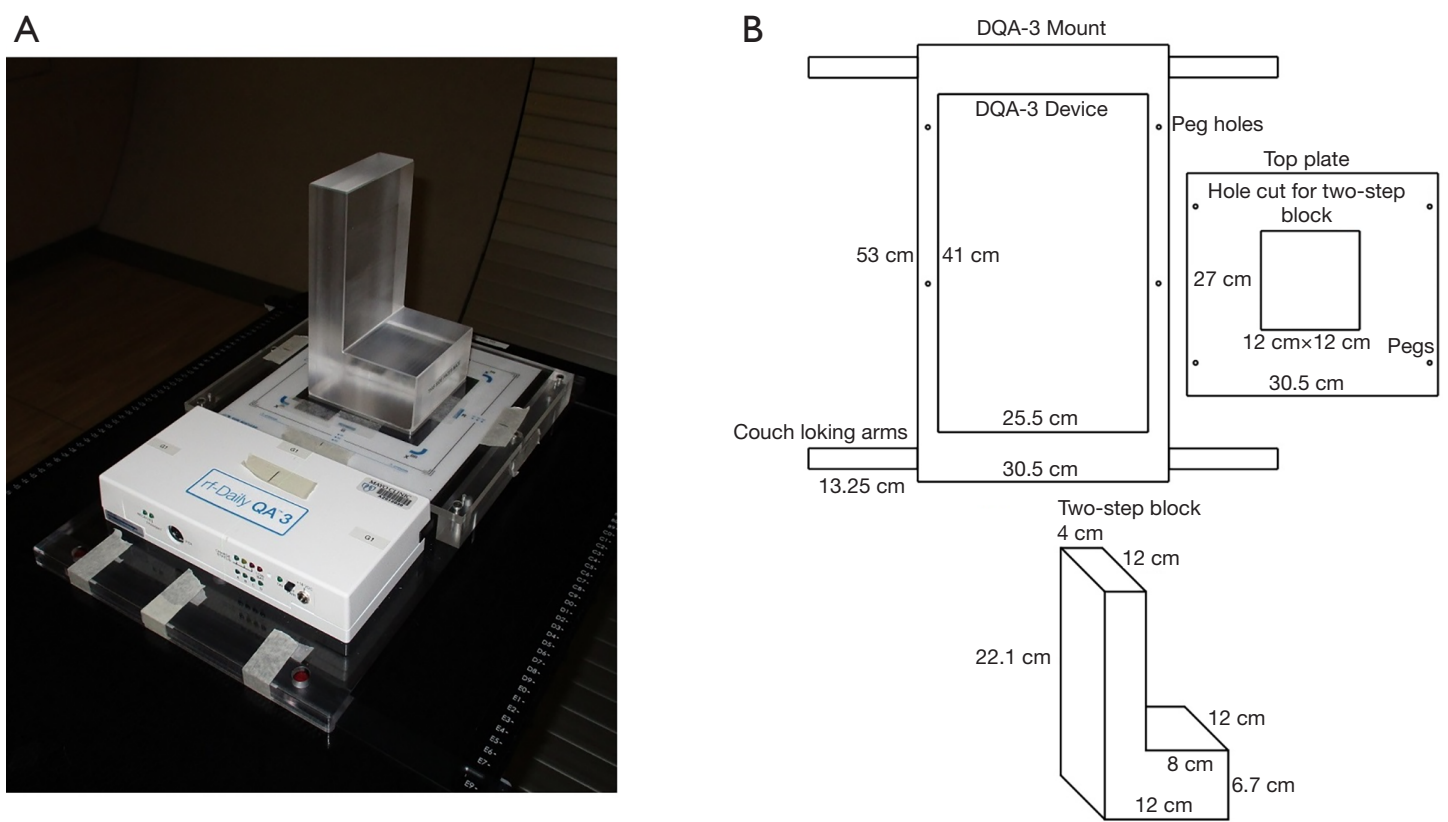

Figure 6 Daily QA apparatus used at Mayo Clinic Arizona, which is based on the procedures described in Younkin et al. (73). (A) The DQA-3 is placed in a mount that is indexed to the table. A single AP treatment field is used to perform all dosimetry tests. (B) Schematic diagrams showing the dimensions of the DQA-3 mount and the two-step phantom. QA, quality assurance; AP, anterior-posterior.

two-step wedge, and a single field for all dosimetry tests (73). Figure 7 shows the dose output of the 4 rooms from April 2017 to August 2020 (40 months in total). There are 3,373 measurement records of output deviation from baseline in total with more than $97.5 \%$ of them within $\pm 1 \%$, well within the $\pm 3 \%$ tolerance recommended by TG-224. Figure 8 shows the distal range deviations of 2 energies (124.8 \& $211.9 \mathrm{MeV}$ ) of one treatment room. These range data were also collected from April 2017 to August 2020. There are 1,732 measurement records of range deviation in total, in which 866 records are from $124.8 \mathrm{MeV}$, as well as another 866 records from $211.9 \mathrm{MeV}$. More than $98.5 \%$ of the range measurement of $124.8 \mathrm{MeV}$ is within $\pm 0.1 \mathrm{~mm}$, and more than $94.5 \%$ of $211.9 \mathrm{MeV}$ is within $\pm 0.2 \mathrm{~mm}$. By comparison, TG-224 recommends a $\pm 1 \mathrm{~mm}$ tolerance for PBS range deviation. Figure 9 shows the spot positions of $99.2 \mathrm{MeV}$ proton beamlets of another treatment room. This set of data was collected between March 2019 to March 2020. The diode triplets used for spot position measurement are located $10 \mathrm{~cm}$ from the isocenter at 4 cardinal directions (left, right, top, and bottom). There are in total 984 measurement records of spot position deviations, in which more than $91 \%$ of the position measurement is within $\pm 0.5 \mathrm{~mm}$.

\section{Discussion and recommendations}

\section{Discussion}

It has been 30 years since the first hospital-based PT facility was opened for cancer treatment. However, procedures for proton daily QA have not yet fully matured. The main factor hampering the development is the very low number of proton treatment rooms compared to conventional radiotherapy. With the emergence of $\mathrm{PBS}$, it is a reasonable expectation that the dosimetric advantages of PT will eventually translate to better clinical outcomes, at least for some tumor sites. This will boost the application of PT for cancer treatment which in turn will increase the demand for commercial solutions for proton daily QA.

The technical challenges of proton dosimetry measurement cannot always be overcome with familiar approaches from conventional radiotherapy. For example, radiation damage from high-energy proton beams can reduce the measurement sensitivity of diode-based systems. It has been reported that the response of p-type diode was decreased $24 \%$ for an accumulated dose of $300 \mathrm{~Gy}$ in conventional radiotherapy (74). It has been observed in our facility that the response of the n-type diode of DQA- 
A

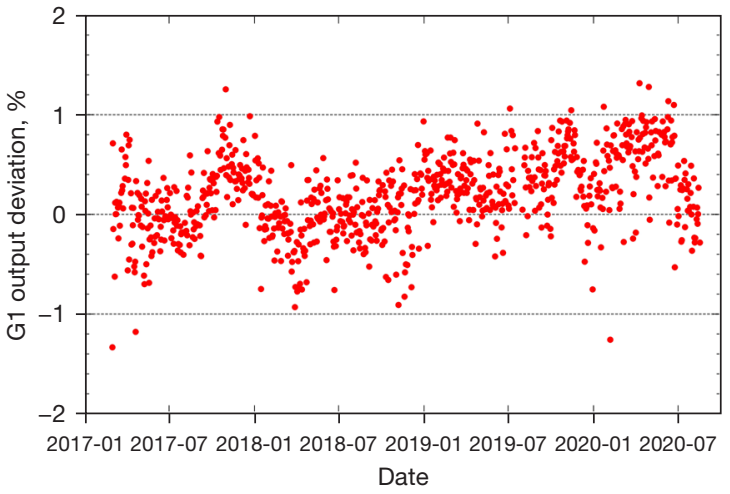

C

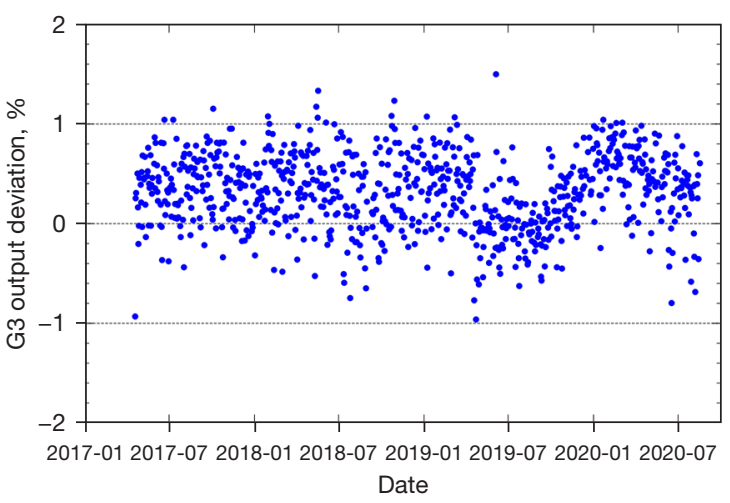

B

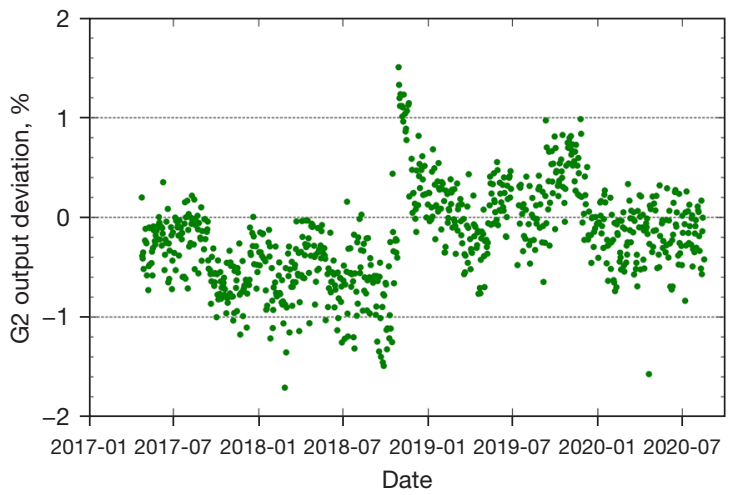

$\mathrm{D}$

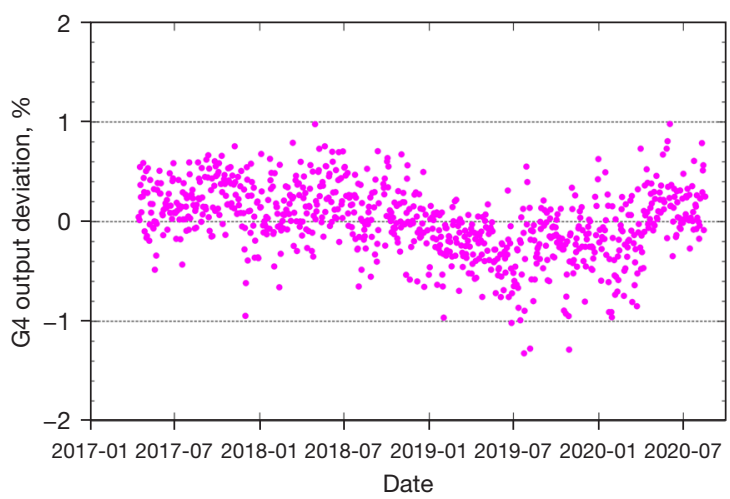

Figure 7 Output deviation measurements from 4 treatment rooms taken over 40 months. There are 3,371 data points in total, in which more than $97.5 \%$ of the data points are within $\pm 1 \%$. (A) Output deviation of G1 gantry room. (B) Output deviation of G2 gantry room. (C) Output deviation of G3 gantry room. (D) Output deviation of G4 gantry room.

3 decreased at the rate of about $5 \%$ per 100 Gy (RBE) (75), which was more than ten times higher than the reported response degradation from photon irradiation (76). The degradation rate was found not to be constant but to decrease with accumulated dose. The response degradation raises the concern about the long-term stability of semiconductor detectors. The proton irradiation of diode detectors might cause even more significant signal degradation (77). Secondly, the proton beamlets usually have widths of less than a centimeter and be deflected laterally up to $20 \mathrm{~cm}$ away from the treatment isocenter. This requires the detector to be able to cover a large area with a millimeter resolution. This requirement presents new challenges to the vendors of linac daily QA equipment, which is not typically designed to check dosimetric parameters in such a setting. Finally, there might be concerns about the proton quenching effect on scintillator detectors and flat-panel detectors due to the linear energy transfer (LET) from proton. The LET of PBS beamlets increases longitudinally in the Bragg peak region (78) and laterally away from the beamlet axis (79). This can lead to an underestimated response from scintillator and flat-panel detectors due to the quenching effect. Though daily QA is a constancy check, the impact of the quenching effect on the baseline value should be quantified and evaluated in the commissioning stage.

With strict tolerances and careful monitoring, a good daily QA program can detect maintenance issues and identify workflow problems before they can affect the clinical practice. Two examples from our institution can be observed in the QA results presented in Figures 7-9. As seen in Figure $7 B$, we identified a fairly sharp, steady drop in G2 daily QA output measurements between April 2017 and November 2018. After first comparing these results to our monthly output measurements to verify this trend, we investigated the G2 gantry and identified a loose connection in a monitor unit chamber that could have led to more severe problems and potentially future downtime. With after-hours repairs and output factor adjustment, 

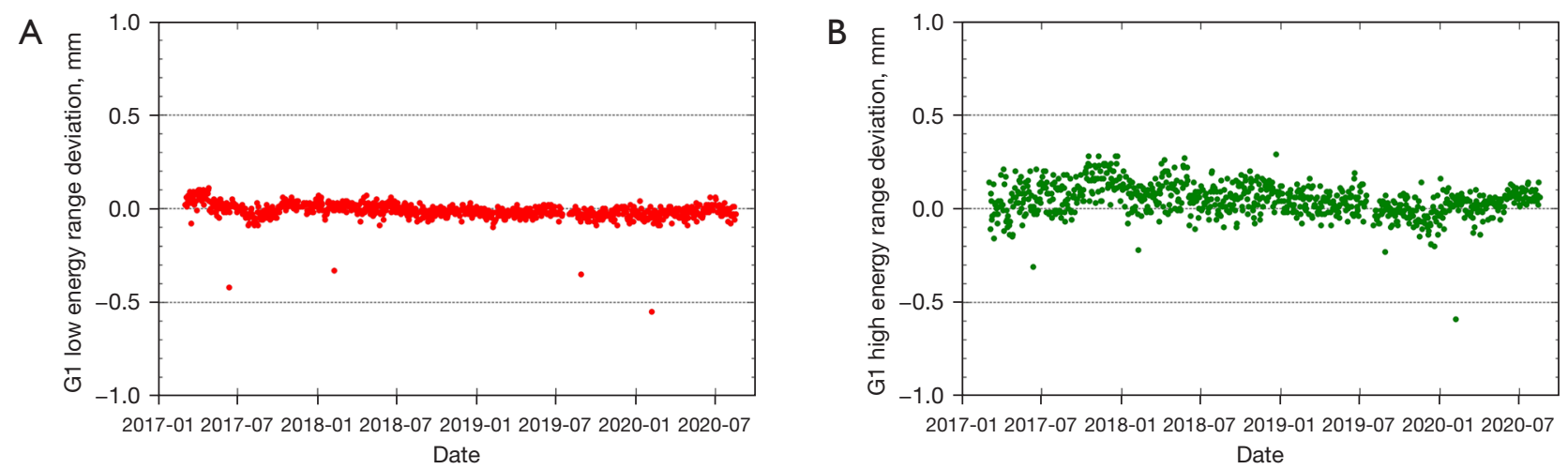

Figure 8 The deviation of distal ranges $(124.8 \& 211.9 \mathrm{MeV})$ of one treatment room over 40 months. There are 1,732 measurement records of range deviation in total, in which 866 records are from $124.8 \mathrm{MeV}$, as well as another 866 records from $124.8 \mathrm{MeV}$. More than $98.5 \%$ of the range measurement of $124.8 \mathrm{MeV}$ is within $\pm 0.1 \mathrm{~mm}$, and more than $94.5 \%$ of $211.9 \mathrm{MeV}$ is within $\pm 0.2 \mathrm{~mm}$. (A) Distal range deviation of 124.8 MeV. (B) Distal range deviation of $211.9 \mathrm{MeV}$.

A

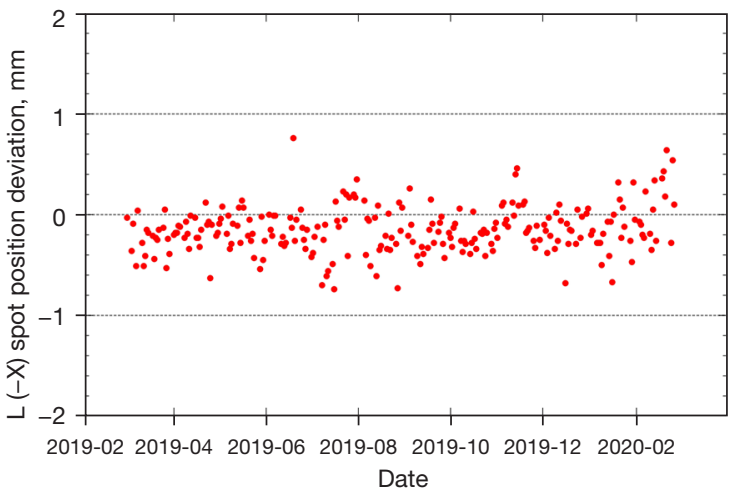

C

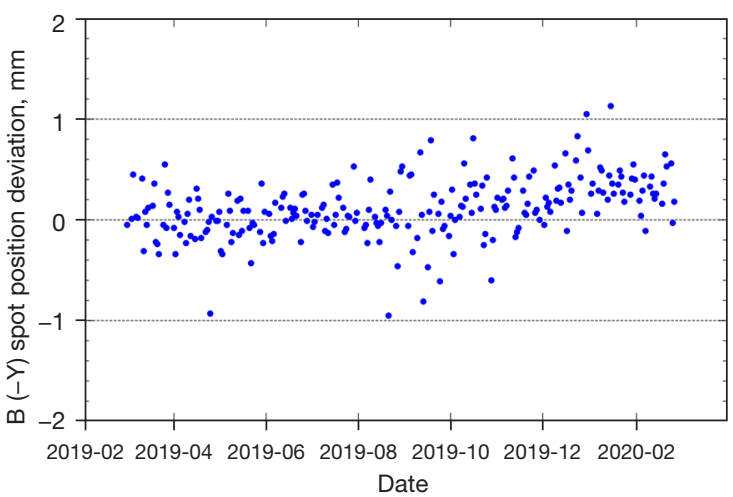

B

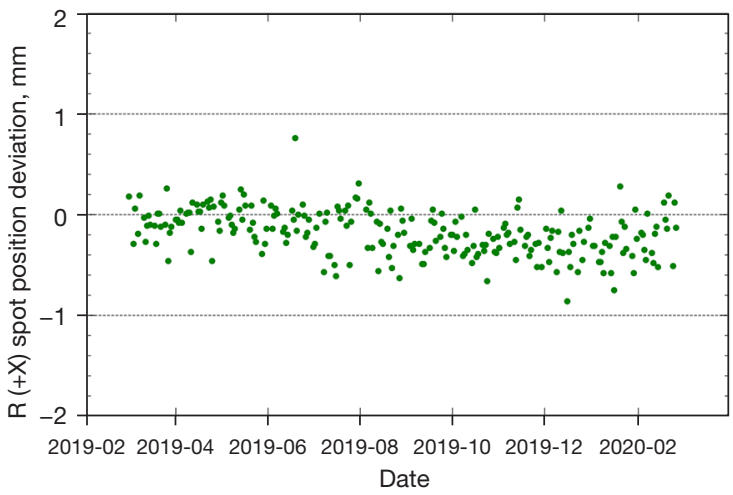

D

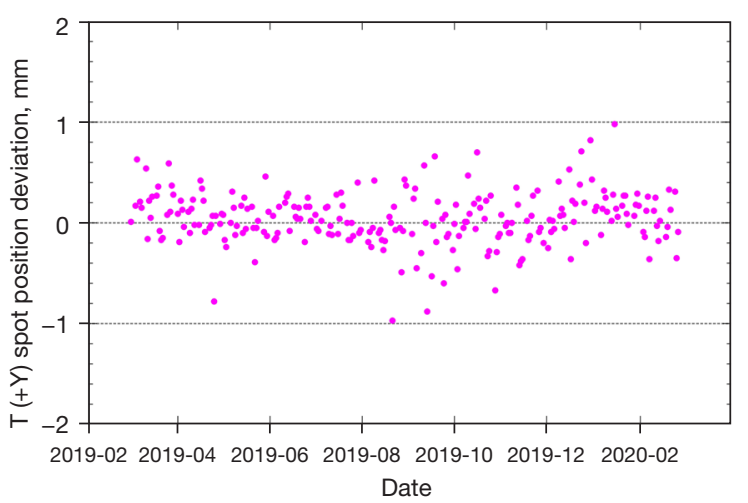

Figure 9 Spot position deviation measurements of the $99.2 \mathrm{MeV}$ proton beamlets of another treatment room taken over 1 year. Measurements in each of the four directions are shown in different colors. There are 984 measurement records of spot positions in total, in which more than $91 \%$ of the position measurement is within $\pm 0.5 \mathrm{~mm}$. (A) Spot position deviation at left diode triplet. (B) Spot position deviation at right diode triplet. (C) Spot position deviation at bottom diode triplet. (D) Spot position deviation at top diode triplet. 
we were able to achieve a more consistent output in G2 without any clinical disruption. Figure $8 A$ has four outliers in the low energy range measurements. High energy range measurements were also low (by approximately similar amounts) on these 4 days. Further investigation revealed that on rare occasions the two-step phantom was slightly tilted in the mount, and the additional tenths of millimeters of path length were detected by our range test. Slight adjustments to the phantom and additional training for therapists corrected this problem.

\section{Recommendations}

Due to evolving technology, uncertainty in QA requirements, and small market share, commercial solutions for proton daily QA will be limited in the foreseeable future. Therefore in-house developed solutions will be the primary choice in the foreseeable future. The hybrid approach of retrofitting commercial devices used in the photon clinic for the needs of proton clinic is expected to be the prudent choice. The hybrid approach is flexible and practical, and enables medical physicists to implement a proton daily QA program tailored to fit the facility configuration and clinical needs. The hybrid solution is also usually cost-effective compared to the vendor solutions available. We recommend that the proton daily QA workflow should include transferring treatment information from the oncology information system (OIS) to the proton machine, moving the couch to the pre-defined setup position, imaging and shifting the couch using the IGRT system, irradiating the QA device to check beam parameters, and sending treatment records back to the OIS. This approach mimics the patient treatment procedures as closely as possible with the daily QA device taking the place of a patient. This gives the best assurances that the proton system will safely and effectively meet the clinical goals for patient treatment every day. However, the hybrid approach takes a substantial time investment to implement as well as some expertise in physics and engineering. This might be challenging for newer, small proton centers due to lack of resources.

We also recommend having therapists carry out proton daily QA rather than MPAs or QMPs. Currently MPAs still perform daily QA in the majority of operational proton treatment centers. Operationally, it is not wise to use the same MPAs in the morning for daily QA before patient treatment and PSQA or other machine QA late in the evening after treatment. On the other hand, the workflow is more smooth if the same therapist runs daily QA and transitions directly into patient treatment after the daily QA results are reviewed by QMPs. This seamless workflow has been proven to greatly streamline clinical workflows (73). However the technical skills required to operate daily QA devices are usually beyond the therapists' professional experience. To prepare therapists to perform daily QA, adequate additional training should be provided. In the case of custom-made QA solutions, special attention should be paid to the simplicity of the hardware and a user-friendly software interface. We also reiterate that regardless of the approach a QMP is required to review the daily QA results and to decide whether the PT equipment is ready for clinical use.

\section{Acknowledgments}

Funding: None.

\section{Footnote}

Conflicts of Interest: All authors have completed the ICMJE uniform disclosure form (available at https://dx.doi. org/10.21037/tro-21-11). XD serves as an unpaid editorial board member of Therapeutic Radiology and Oncology from March 2021 to February 2023. Dr. WL reports grants from Arizona Biomedical Research Commissioning, grants from the Lawrence W. and Marilyn W. Matteson Fund for Cancer Research, grants from the Kemper Marley Foundation, outside the submitted work; Dr. WL has 4 US patents (either issued or pending). In addition, Dr. WL has a patent, an accurate and efficient hybrid method based on ray casting to calculate physical dose and linear energy transfer (LET) distribution for intensity-modulated proton therapy with royalties paid to Decimal LLC. The other authors have no conflicts of interest to declare.

Ethical Statement: The authors are accountable for all aspects of the work in ensuring that questions related to the accuracy or integrity of any part of the work are appropriately investigated and resolved.

Open Access Statement: This is an Open Access article distributed in accordance with the Creative Commons Attribution-NonCommercial-NoDerivs 4.0 International License (CC BY-NC-ND 4.0), which permits the noncommercial replication and distribution of the article with the strict proviso that no changes or edits are made and the original work is properly cited (including links to both the formal publication through the relevant DOI and the license). 
See: https://creativecommons.org/licenses/by-nc-nd/4.0/.

\section{References}

1. Paganetti H, Beltran CJ, Both S, et al. Roadmap: proton therapy physics and biology. Phys Med Biol 2020. doi: 10.1088/1361-6560/abcd16.

2. Wilson RR. Radiological use of fast protons. Radiology 1946;47:487-91.

3. Quan EM, Liu W, Wu R, et al. Preliminary evaluation of multifield and single-field optimization for the treatment planning of spot-scanning proton therapy of head and neck cancer. Med Phys 2013;40:081709.

4. Frank SJ, Cox JD, Gillin M, et al. Multifield optimization intensity modulated proton therapy for head and neck tumors: a translation to practice. Int J Radiat Oncol Biol Phys 2014;89:846-53.

5. Arjomandy B, Taylor P, Ainsley C, et al. AAPM task group 224: Comprehensive proton therapy machine quality assurance. Med Phys 2019;46:e678-705.

6. MacKay RI. Image Guidance for Proton Therapy. Clin Oncol (R Coll Radiol) 2018;30:293-8.

7. Coutrakon GB. Accelerators for heavy-charged-particle radiation therapy. Technol Cancer Res Treat 2007;6:49-54.

8. Peach K, Wilson P, Jones B. Accelerator science in medical physics. Br J Radiol 2011;84 Spec No 1:S4-10.

9. Smith A, Gillin M, Bues M, et al. The M. D. Anderson proton therapy system. Med Phys 2009;36:4068-83.

10. Farr JB, Mascia AE, Hsi WC, et al. Clinical characterization of a proton beam continuous uniform scanning system with dose layer stacking. Med Phys 2008;35:4945-54.

11. Gillin MT, Sahoo N, Bues M, et al. Commissioning of the discrete spot scanning proton beam delivery system at the University of Texas M.D. Anderson Cancer Center, Proton Therapy Center, Houston. Med Phys 2010;37:154-63.

12. Zhang $\mathrm{X}$, Li $\mathrm{Y}$, Pan $\mathrm{X}$, et al. Intensity-modulated proton therapy reduces the dose to normal tissue compared with intensity-modulated radiation therapy or passive scattering proton therapy and enables individualized radical radiotherapy for extensive stage IIIB non-small-cell lung cancer: a virtual clinical study. Int J Radiat Oncol Biol Phys 2010;77:357-66.

13. Schild SE, Rule WG, Ashman JB, et al. Proton beam therapy for locally advanced lung cancer: A review. World J Clin Oncol 2014;5:568-75.

14. Nichiporov D, Hsi W, Farr J. Beam characteristics in two different proton uniform scanning systems: a side-by-side comparison. Med Phys 2012;39:2559-68.

15. Lomax AJ. Intensity modulated proton therapy and its sensitivity to treatment uncertainties 2 : the potential effects of inter-fraction and inter-field motions. Phys Med Biol 2008;53:1043-56.

16. Bert C, Durante M. Motion in radiotherapy: particle therapy. Phys Med Biol 2011;56:R113-44.

17. Lambert J, Suchowerska N, McKenzie DR, et al. Intrafractional motion during proton beam scanning. Phys Med Biol 2005;50:4853-62.

18. Phillips MH, Pedroni E, Blattmann H, et al. Effects of respiratory motion on dose uniformity with a charged particle scanning method. Phys Med Biol 1992;37:223-34.

19. Pedroni E, Scheib S, Böhringer T, et al. Experimental characterization and physical modelling of the dose distribution of scanned proton pencil beams. Phys Med Biol 2005;50:541-61.

20. Ciocca M, Magro G, Mastella E, et al. Design and commissioning of the non-dedicated scanning proton beamline for ocular treatment at the synchrotron-based CNAO facility. Med Phys 2019;46:1852-62.

21. Liu W, Zhang X, Li Y, et al. Robust optimization of intensity modulated proton therapy. Med Phys 2012;39:1079-91.

22. Liu W, Frank SJ, Li X, et al. Effectiveness of robust optimization in intensity-modulated proton therapy planning for head and neck cancers. Med Phys 2013;40:051711.

23. Liu W, Frank SJ, Li X, et al. PTV-based IMPT optimization incorporating planning risk volumes vs robust optimization. Med Phys 2013;40:021709.

24. Liu W, Li Y, Li X, et al. Influence of robust optimization in intensity-modulated proton therapy with different dose delivery techniques. Med Phys 2012;39:3089-101.

25. Liu W, Mohan R, Park P, et al. Dosimetric benefits of robust treatment planning for intensity modulated proton therapy for base-of-skull cancers. Pract Radiat Oncol 2014;4:384-91.

26. Liu C, Bhangoo RS, Sio TT, et al. Dosimetric comparison of distal esophageal carcinoma plans for patients treated with small-spot intensity-modulated proton versus volumetric-modulated arc therapies. J Appl Clin Med Phys 2019;20:15-27.

27. Liu C, Yu NY, Shan J, et al. Technical Note: Treatment planning system (TPS) approximations matter - comparing intensity-modulated proton therapy (IMPT) plan quality and robustness between a commercial and an in-house 
developed TPS for nonsmall cell lung cancer (NSCLC). Med Phys 2019;46:4755-62.

28. Liu C, Schild SE, Chang JY, et al. Impact of Spot Size and Spacing on the Quality of Robustly Optimized Intensity Modulated Proton Therapy Plans for Lung Cancer. Int J Radiat Oncol Biol Phys 2018;101:479-89.

29. Liu W, Liao Z, Schild SE, et al. Impact of respiratory motion on worst-case scenario optimized intensity modulated proton therapy for lung cancers. Pract Radiat Oncol 2015;5:e77-86.

30. Chen W, Unkelbach J, Trofimov A, et al. Including robustness in multi-criteria optimization for intensity-modulated proton therapy. Phys Med Biol 2012;57:591-608.

31. Fredriksson A, Forsgren A, Hårdemark B. Minimax optimization for handling range and setup uncertainties in proton therapy. Med Phys 2011;38:1672-84.

32. Unkelbach J, Alber M, Bangert $M$, et al. Robust radiotherapy planning. Phys Med Biol 2018;63:22TR02.

33. Unkelbach J, Bortfeld T, Martin BC, et al. Reducing the sensitivity of IMPT treatment plans to setup errors and range uncertainties via probabilistic treatment planning. Med Phys 2009;36:149-63.

34. Unkelbach J, Chan TC, Bortfeld T. Accounting for range uncertainties in the optimization of intensity modulated proton therapy. Phys Med Biol 2007;52:2755-73.

35. An Y, Liang J, Schild SE, et al. Robust treatment planning with conditional value at risk chance constraints in intensity-modulated proton therapy. Med Phys 2017;44:28-36.

36. Shan J, An Y, Bues M, et al. Robust optimization in IMPT using quadratic objective functions to account for the minimum MU constraint. Med Phys 2018;45:460-9.

37. Shan J, Sio TT, Liu C, et al. A novel and individualized robust optimization method using normalized dose interval volume constraints (NDIVC) for intensity-modulated proton radiotherapy. Med Phys 2019;46:382-93.

38. Inaniwa $T$, Kanematsu $\mathrm{N}$, Furukawa $\mathrm{T}$, et al. A robust algorithm of intensity modulated proton therapy for critical tissue sparing and target coverage. Phys Med Biol 2011;56:4749-70.

39. Feng H, Shan J, Ashman JB, et al. Technical Note: 4D robust optimization in small spot intensity-modulated proton therapy (IMPT) for distal esophageal carcinoma. Med Phys 2021;48:4636-47.

40. Tryggestad EJ, Liu W, Pepin MD, et al. Managing treatment-related uncertainties in proton beam radiotherapy for gastrointestinal cancers. J Gastrointest
Oncol 2020;11:212-24.

41. Yu J, Zhang X, Liao L, et al. Motion-robust intensitymodulated proton therapy for distal esophageal cancer. Med Phys 2016;43:1111-8.

42. Siregar H, Bäumer C, Blanck O, et al. Mitigation of motion effects in pencil-beam scanning - Impact of repainting on $4 \mathrm{D}$ robustly optimized proton treatment plans for hepatocellular carcinoma. Z Med Phys 2020. doi: 10.1016/j.zemedi.2020.08.001.

43. Chang JY, Zhang X, Knopf A, et al. Consensus Guidelines for Implementing Pencil-Beam Scanning Proton Therapy for Thoracic Malignancies on Behalf of the PTCOG Thoracic and Lymphoma Subcommittee. Int J Radiat Oncol Biol Phys 2017;99:41-50.

44. Engwall E, Fredriksson A, Glimelius L. 4D robust optimization including uncertainties in time structures can reduce the interplay effect in proton pencil beam scanning radiation therapy. Med Phys 2018. doi: 10.1002/mp.13094.

45. Liu W, Patel SH, Shen JJ, et al. Robustness quantification methods comparison in volumetric modulated arc therapy to treat head and neck cancer. Pract Radiat Oncol 2016;6:e269-75.

46. Park PC, Cheung JP, Zhu XR, et al. Statistical assessment of proton treatment plans under setup and range uncertainties. Int $\mathrm{J}$ Radiat Oncol Biol Phys 2013;86:1007-13.

47. Trofimov A, Unkelbach J, DeLaney TF, et al. Visualization of a variety of possible dosimetric outcomes in radiation therapy using dose-volume histogram bands. Pract Radiat Oncol 2012;2:164-71.

48. Liu W, Patel SH, Harrington DP, et al. Exploratory study of the association of volumetric modulated arc therapy (VMAT) plan robustness with local failure in head and neck cancer. J Appl Clin Med Phys 2017;18:76-83.

49. Shan J, Yang Y, Schild SE, et al. Intensity-modulated proton therapy (IMPT) interplay effect evaluation of asymmetric breathing with simultaneous uncertainty considerations in patients with non-small cell lung cancer. Med Phys 2020;47:5428-40.

50. Matney J, Park PC, Bluett J, et al. Effects of respiratory motion on passively scattered proton therapy versus intensity modulated photon therapy for stage III lung cancer: are proton plans more sensitive to breathing motion? Int J Radiat Oncol Biol Phys 2013;87:576-82.

51. Younkin JE, Morales DH, Shen J, et al. Technical Note: Multiple energy extraction techniques for synchrotronbased proton delivery systems may exacerbate motion interplay effects in lung cancer treatments. Med Phys 
2021;48:4812-23.

52. Matney JE, Park PC, Li H, et al. Perturbation of waterequivalent thickness as a surrogate for respiratory motion in proton therapy. J Appl Clin Med Phys 2016;17:368-78.

53. Younkin JE, Bues M, Sio TT, et al. Multiple energy extraction reduces beam delivery time for a synchrotronbased proton spot-scanning system. Adv Radiat Oncol 2018;3:412-20.

54. Verhey LJ, Goitein M, McNulty P, et al. Precise positioning of patients for radiation therapy. Int J Radiat Oncol Biol Phys 1982;8:289-94.

55. Landry G, Hua CH. Current state and future applications of radiological image guidance for particle therapy. Med Phys 2018;45:e1086-95. Erratum in: Med Phys 2019;46:1088.

56. Bissonnette JP, Balter PA, Dong L, et al. Quality assurance for image-guided radiation therapy utilizing CT-based technologies: a report of the AAPM TG-179. Med Phys 2012;39:1946-63.

57. Klein EE, Hanley J, Bayouth J, et al. Task Group 142 report: quality assurance of medical accelerators. Med Phys 2009;36:4197-212.

58. Fontenot JD, Alkhatib H, Garrett JA, et al. AAPM Medical Physics Practice Guideline 2.a: Commissioning and quality assurance of X-ray-based image-guided radiotherapy systems. J Appl Clin Med Phys 2014;15:4528.

59. Kutcher GJ, Coia L, Gillin M, et al. Comprehensive QA for radiation oncology: report of AAPM Radiation Therapy Committee Task Group 40. Med Phys 1994;21:581-618.

60. Slater JD. Development and operation of the Loma Linda University Medical Center proton facility. Technol Cancer Res Treat 2007;6:67-72.

61. Hernandez Morales D, Shan J, Liu W, et al. Automation of routine elements for spot-scanning proton patientspecific quality assurance. Med Phys 2019;46:5-14.

62. Johnson JE, Beltran C, Wan Chan Tseung H, et al. Highly efficient and sensitive patient-specific quality assurance for spot-scanned proton therapy. PLoS One 2019;14:e0212412.

63. Meijers A, Guterres Marmitt G, Ng Wei Siang K, et al. Feasibility of patient specific quality assurance for proton therapy based on independent dose calculation and predicted outcomes. Radiother Oncol 2020;150:136-41.

64. Rana S, Bennouna J, Samuel EJJ, et al. Development and long-term stability of a comprehensive daily QA program for a modern pencil beam scanning (PBS) proton therapy delivery system. J Appl Clin Med Phys 2019;20:29-44.
65. Su Z, Hsi W, Forthomme J, et al. Evaluations of a flatpanel based compact daily quality assurance device for proton pencil beam scanning (PBS) system. Phys Med 2020;80:243-50.

66. Actis O, Meer D, König S, et al. A comprehensive and efficient daily quality assurance for PBS proton therapy. Phys Med Biol 2017;62:1661-75.

67. Arjomandy B, Sahoo N, Zhu XR, et al. An overview of the comprehensive proton therapy machine quality assurance procedures implemented at The University of Texas $M$. D. Anderson Cancer Center Proton Therapy CenterHouston. Med Phys 2009;36:2269-82.

68. Poenisch F, Sahoo N, Li H, et al. Physics Quality Assurance. In: Frank SJ, Zhu XR. Proton Therapy E-Book: Indications, Techniques, and Outcomes. Philadelphia: Elsevier Health Sciences, 2020:80-105.

69. Bizzocchi N, Fracchiolla F, Schwarz M, et al. A fast and reliable method for daily quality assurance in spot scanning proton therapy with a compact and inexpensive phantom. Med Dosim 2017;42:238-46.

70. Shi C, Chen Q, Yu F, et al. Auto-Trending daily quality assurance program for a pencil beam scanning proton system aligned with TG 224. J Appl Clin Med Phys 2021;22:117-27.

71. Ding X, Zheng Y, Zeidan O, et al. A novel daily QA system for proton therapy. J Appl Clin Med Phys 2013;14:4058.

72. Lambert J, Bäumer C, Koska B, et al. Daily QA in proton therapy using a single commercially available detector. J Appl Clin Med Phys 2014;15:5005.

73. Younkin JE, Shen J, Bues M, et al. Technical Note: An efficient daily QA procedure for proton pencil beam scanning. Med Phys 2018;45:1040-9.

74. Pacilio M, De Angelis C, Onori S, et al. Characteristics of silicon and diamond detectors in a $60 \mathrm{MeV}$ proton beam. Phys Med Biol 2002;47:N107-12.

75. Younkin JE, Robertson DG, Liu W, et al. Technical Note: Long-term monitoring of diode sensitivity degradation induced by proton irradiation. Med Phys 2021;48:6634-41.

76. Jursinic PA, Nelms BE. A 2-D diode array and analysis software for verification of intensity modulated radiation therapy delivery. Med Phys 2003;30:870-9.

77. Zou M, Bohon J, Smedley J, et al. Proton radiation effects on carrier transport in diamond radiation detectors. AIP Adv 2020;10:025004.

78. Romano F, Cirrone GA, Cuttone G, et al. A Monte Carlo study for the calculation of the average linear energy transfer (LET) distributions for a clinical proton beam line and a radiobiological carbon ion beam line. Phys Med Biol 
2014;59:2863-82.

79. Deng W, Ding X, Younkin JE, et al. Hybrid 3D analytical linear energy transfer calculation algorithm based on

doi: $10.21037 /$ tro-21-11

Cite this article as: Ding X, Younkin JE, Shen J, Bues M, Liu $W$. A critical review of the practices of proton daily quality assurance programs. Ther Radiol Oncol 2021;5:22. precalculated data from Monte Carlo simulations. Med Phys 2020;47:745-52. 